\title{
Finite element method analysis of band gap and transmission of two-dimensional metallic photonic crystals at terahertz frequencies
}

\author{
Elif Degirmenci and Pascal Landais* \\ Radio and Optical Communications Laboratory, The RINCE Institute, Dublin City University, Glasnevin, Dublin 9, Ireland \\ ${ }^{*}$ Corresponding author: landaisp@eeng.dcu.ie
}

Received 23 May 2013; revised 18 August 2013; accepted 25 September 2013;

posted 25 September 2013 (Doc. ID 190347); published 18 October 2013

\begin{abstract}
Photonic band gap and transmission characteristics of 2D metallic photonic crystals at $\mathrm{THz}$ frequencies have been investigated using finite element method (FEM). Photonic crystals composed of metallic rods in air, in square and triangular lattice arrangements, are considered for transverse electric and transverse magnetic polarizations. The modes and band gap characteristics of metallic photonic crystal structure are investigated by solving the eigenvalue problem over a unit cell of the lattice using periodic boundary conditions. A photonic band gap diagram of dielectric photonic crystal in square lattice array is also considered and compared with well-known plane wave expansion results verifying our FEM approach. The photonic band gap designs for both dielectric and metallic photonic crystals are consistent with previous studies obtained by different methods. Perfect match is obtained between photonic band gap diagrams and transmission spectra of corresponding lattice structure. () 2013 Optical Society of America

OCIS codes: (160.5293) Photonic bandgap materials; (160.5298) Photonic crystals; (160.3900)

Metals; (040.2235) Far infrared or terahertz.

http://dx.doi.org/10.1364/AO.52.007367
\end{abstract}

\section{Introduction}

Terahertz $(\mathrm{THz})$ waves refer to electromagnetic radiation that has a frequency between 0.3 and $10 \mathrm{THz}$. $\mathrm{THz}$ radiation lies between microwave and infrared regions and corresponds to the submillimeter and far infrared wavelength range. In recent years, this region has become scientifically available and attracted a lot of attention on its potential and promising applications in many areas such as sensing, imaging, security, spectroscopy, and telecommunications [1-3]. With the rise of $\mathrm{THz}$-frequency-based technology and developments in generating $\mathrm{THz}$ radiation and detection, the need for compact, functional optical components for the $\mathrm{THz}$ spectral range has increased.

$1559-128 X / 13 / 307367-09 \$ 15.00 / 0$

(C) 2013 Optical Society of America
For the last few decades, photonic crystals have attracted a great deal of attention due to their capability to control and manipulate electromagnetic wave propagation within photonic band gaps determined by certain frequency ranges and directions [4]. They have become the subject of both theoretical and experimental studies in optoelectronic and integrated optics because of their low losses and low dispersion properties.

Photonic crystals are good candidates to construct a variety of components for compact application systems operating at $\mathrm{THz}$ frequencies such as waveguides [5], THz switches [6], filters [7], splitters [] etc. with small sizes, low loss, and flexibility of fabrication due to comparatively large $\mathrm{THz}$ wavelengths. Compared to dielectric photonic crystals, metallic photonic crystals offer much wider photonic band gaps, which leads to wider frequency ranges [9-11]. Metallic photonic crystals are more reflective 
than their dielectric counterparts $[12,13]$. Therefore, it is possible to design more compact and lighter devices since fewer periods with metallic photonic crystals are required $[14,15]$. Moreover, metallic photonic crystals are more suited to handling higher-energy $\mathrm{THz}$ signals than dielectric photonic crystals. Studies of metallic photonic crystals have mostly concentrated in the microwave and millimeter frequency range due to their low propagation losses $[9,16,17]$. In the $\mathrm{THz}$ range, the losses caused by finite conductivity of metals are comparatively smaller than those at optical frequencies. They have also been used in the $\mathrm{THz}$ frequency range to study their filtering and wave guiding capability [18-23].

The band gap diagram, namely dispersion diagram, is achieved by solving the eigenvalue problem. Dispersion diagrams are key to determining band gaps and pass bands of periodic structures that provide information for designing an optoelectronic device. Many numerical methods, including the plane wave expansion (PWE), finite difference time domain (FDTD), finite difference (FD), finite element, etc. have been utilized for analyzing the photonic band gap structures and achieving the band gap diagram. Among these approaches, the conventional PWE method is the most used and reliable method for nondispersive materials. However, this approach has a limitation when it comes to metallic photonic crystal structure. The equations for the conventional PWE method are valid for real-valued dielectric constant. Consequently, conventional PWE method cannot deal with lossy materials since the loss in a material is incorporated into the model through the imaginary part of the dielectric constant. In order to calculate a band gap diagram of photonic crystal with metallic inclusions, a modified/extended PWE method is proposed [24,25].

The photonic band gap of metallic photonic crystals has been theoretically studied by many methods that consider photonic crystals with frequencydependent dielectric constants. A 2D array of infinitely long metallic cylinders embedded in vacuum was carried out by Kuzmiak et al. in 1994 [24]. The modified PWE method calculates the eigenvalues to study the band gap diagrams of metallic photonic crystals with dielectric constant in free-electron form arranged in square and triangular patterns. The permittivity is real-valued so the absorption is not taken into account. This study showed the significant differences in the band structures for TM and TE modes. For TM mode, metallic photonic crystals exhibit a broad photonic band gap between zero frequency and their cutoff frequency. On the other hand, for TE mode, metallic photonic crystals produce flat bands with very low group velocities. Another study was carried out by using the generalized Rayleigh identity method to calculate band diagrams of $2 \mathrm{D}$ arrays of perfectly conducting cylinders [26]. Later, the photonic bands of metallic systems by means of the numerical simulation of the dipole radiation based on the FDTD method was carried out in
2001 by Sakoda and co-workers [27,28]. The results were in agreement with Kuzmiak's study; moreover, the flat bands with very low group velocities seen in TE mode were explained by the existence of plasmon resonances. For these modes, wave propagation is provided with plasmon resonances rather than a band gap mechanism. Some other approaches are used to calculate the photonic band gap of metallic photonic crystals. They include transfer matrix method [29], FDTD method [10,30], finite difference method $[13,31]$, finite difference frequency domain method [32], multiple multipole method [33], Korringa-Kohn-Rostoker method [34,35], multiple scattering methods [36-37], and order-N method [38].

In this paper, the photonic band structure and transmission calculations of $2 \mathrm{D}$ metallic photonic crystals in the $\mathrm{THz}$ frequencies are investigated using finite element method (FEM). The FEM has proven to be a very reliable and effective numerical approach for modeling and simulating a wide range of physics and multiphysics problems, especially for complex structures. It is also able to resolve and describe wave propagation as well as the dispersion diagram of photonic crystal structures [39-41]. We implement calculations using a commercially available software based on FEM [42].

Photonic crystals consisting of metallic cylinders in air arranged by two types of lattice arrays, namely square and triangular lattice structures, are considered. First, the FEM approach is introduced for the calculation of $2 \mathrm{D}$ photonic crystal bands for metallic photonic crystals considering dispersive properties. In the calculations, frequency dependence and losses are considered by specifying the material properties with the Drude model [43]. We calculate the band gap structure of metallic photonic crystals by solving nonlinear quadratic eigenvalue equations using the FEM approach for two polarization directions, transverse electric (TE) and transverse magnetic (TM). In order to verify our FEM approach, FEM and PWE methods are compared for nondispersive photonic crystal. The calculated results are presented in Section 2. In Section 3, THz transmission properties of metallic photonic crystals are investigated and compared to the band gap diagram of corresponding crystal structures, and finally conclusions are given in Section $\underline{4}$.

\section{Computation of Photonic Band Gaps}

Electromagnetic wave propagation in a photonic crystal structure can be studied by solving Maxwell's Equations in the following form for magnetic field [4]:

$$
\nabla \times\left(\frac{1}{\varepsilon(\vec{r})} \nabla \times H(\vec{r})\right)=\left(\frac{\omega}{c}\right)^{2} H(\vec{r}),
$$

where $\omega$ is the angular frequency, $c$ is the speed of light in vacuum, and $\varepsilon(r)$ is the relative permittivity of material. 
We consider that the $2 \mathrm{D}$ photonic crystals system is periodic in the $x$ and $y$ direction and homogeneous in the $z$ direction. The modes in 2D photonic crystals can be classified into two polarizations: the TM mode and the TE mode. For the TE mode, the magnetic field is along the $z$ direction $\left(\vec{H}=H_{z} \hat{a}_{z}\right)$ and for the TM mode, it is the electric field $\left(\vec{E}=E_{z} \hat{a}_{z}\right)$.

The $2 \mathrm{D}$ photonic crystal is a medium described in terms of $\varepsilon$. As the medium is periodic, $\varepsilon(\vec{r})=\varepsilon(\vec{r}+\vec{R})$, where $\vec{R}$ is the lattice translation vector. The translational symmetry of the dielectric function implies that Bloch waves are determined from a problem over one cell in the lattice array. According to Bloch theorem, the wave function can be written as the product of a periodic function where $u(\vec{r})=u(\vec{r}+\vec{R})$ and an exponential factor

$$
H(\vec{r})=u(\vec{r}) e^{-i \vec{k} \cdot \vec{r}},
$$

where $\vec{k}=\left(k_{x}+k_{y}\right)$ is the wavevector of the BlochFloquet wave that lies within the Brillouin zone. Electric or magnetic field then can be determined by solving the eigenvalue problem with the wavevector $\vec{k}$ or the frequency $\omega$ with the unknowns of Eq. (1).

Most computation methods solve eigenvalue equations by specifying Bloch $k$ vectors and computing frequencies $\omega$ as a function of the wavenumber $\omega(\vec{k})$. This is very convenient for photonic crystals consisting of nondispersive materials where the dielectric constant is frequency independent since the problem reduces to a generalized linear eigenvalue equation. However, when dealing with dispersive material such as metals, the eigenvalue problem becomes nonlinear and iterative algorithms are required to reach a solution [44].

For the calculations of the dispersion relation calculations, we use FEM by specifying the frequency $\omega$ and solving the eigenvalue equations for wave vectors $\vec{k}$ obtaining the bands as $\vec{k}(\omega)$. Material dispersion and frequency dependency are considered since the frequency is the parameter of the eigenvalue equation. With the formulation developed by Hiett, solving the eigenvalue equation considering $k$ as the eigenvalue instead of the frequency results in a more tractable quadratic eigenvalue equation in $k$ which can be solved by proper linearization procedures $[45,46]$. Moreover, the calculated eigenvalues are complex-valued when dispersive/lossy material is considered.

The propagating modes in a photonic crystal are described by real-valued wave vector, while the imaginary part of the complex wave vector exhibits the evanescent modes. Therefore, this method is more convenient for photonic crystal containing dispersive material such as metals.

In order to obtain band structure, quadratic eigenvalue calculations are performed over a unit cell of a photonic crystal lattice with Floquet periodic boundary conditions. The computational domain is

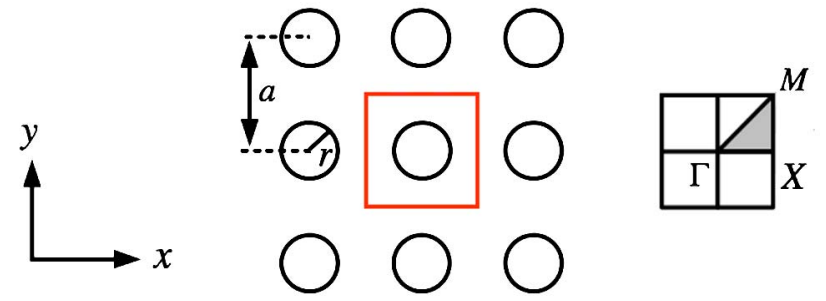

Fig. 1. Square lattice with unit cell is highlighted with red center square. Brillouin zone is shown in the shaded area, indicating the high-symmetry points $\Gamma, X$, and $M$, which are the edges of the Brillouin zone.

discretized by means of FEM, turning the nonlinear eigenvalue equation into an algebraic linear equation system. Bloch $k$ vectors are computed along specific $k$ direction.

The FEM calculations are carried out on the unit cell as seen in Figs. 1 and 2 , applying the Floquet periodic boundary conditions on exterior boundaries of the unit cell that extend the structure to infinity. Figures 1 and 2 show the top view of the square lattice and triangular lattice arrays, respectively, with radius $r$ and lattice spacing $a$. The Brillouin zones are shown in shaded areas, indicating the high symmetry points.

Table 1 shows the reciprocal lattice vectors in the Brillouin zone used for calculation. Photonic bands are calculated along high-symmetry crystal directions over the entire irreducible Brillouin zone.

\section{A. Validation of FEM Model}

Here, we solve eigenvalue calculations for band structures of the 2D dielectric photonic crystals. The FEM calculations are verified by comparing the results to the well-known PWE method studying a dielectric material, since the PWE method cannot be applicable for materials with losses, e.g., metals. Dispersion relation of square lattice pattern is studied for TE and TM polarizations.

2D photonic crystal structures consist of silicon ( $\varepsilon=11.7$ ) circular cylinders with radius $r=0.2 a \mathrm{em}$ bedded in air $(\varepsilon=1)$ background as shown schematically in Fig. 1. The Si cylinders form a square lattice array with lattice spacing $(a=50 \mu \mathrm{m})$. Light propagation is considered in the $x y$ plane of the square lattice structure.

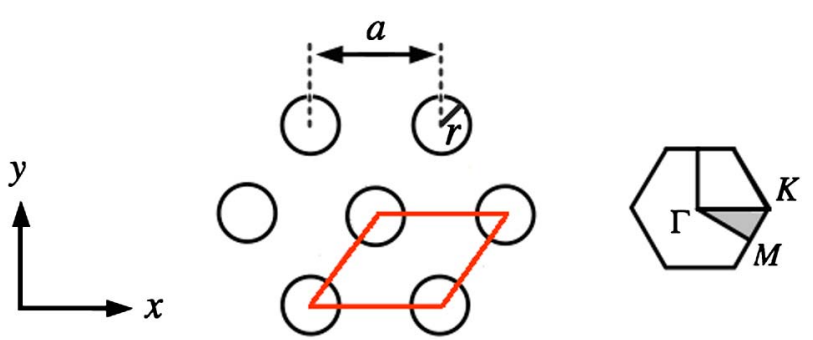

Fig. 2. Triangular lattice with unit cell is highlighted with red parallelogram. Brillouin zone is shown in shaded area, indicating the high-symmetry points $\Gamma, K$, and $M$, which are the edges of Brillouin zone. 
Table 1. Symbols and Wave Vector Directions for Square and Triangular Lattices

\begin{tabular}{lccc}
\hline \multicolumn{2}{c}{ Square Lattice } & & \multicolumn{2}{c}{ Triangular Lattice } \\
\hline Symbol & Reciprocal Lattice & Symbol & Reciprocal Lattice \\
\hline$\Gamma$ & $k=0$ & $\Gamma$ & $k=0$ \\
$X$ & $k=\left((\pi / a) \hat{a}_{x}\right)$ & $M$ & $k=(2 \pi / \sqrt{3} a) \hat{a}_{y}$ \\
$M$ & $k=(\pi / a) \hat{a}_{x}+(\pi / a) \hat{a}_{y}$ & $K$ & $k=(2 \pi / 3 a) \hat{a}_{x}+(2 \pi / \sqrt{3} a) \hat{a}_{y}$ \\
\hline
\end{tabular}

Figures $\underline{3}$ and $\underline{4}$ present the band gap diagrams along the high symmetry points $\Gamma, X$, and $M$ of the Brillouin zone for TM and TE modes, respectively. The solid black lines represent the bands calculated using PWE method and the red dots, the bands calculated using FEM method. As can be seen from Fig. 3, there is a large band gap for TM mode in the normalized frequency range between 0.28 and 0.42 and a smaller band gap between 0.72 and 0.75 . In TE mode, no band gap appears for this configuration, as can be seen in Fig. 4. For both band gap calculation in TM and TE mode, FEM results match almost perfectly with the results produced by the PWE method [47]. This study has validated our FEM approach; in the next section our simulations are adapted to metallic photonic crystals.

\section{B. Metallic Photonic Band Gaps}

Drude model is utilized to calculate the complex, frequency-dependent dielectric function for metallic material in the form [43]

$$
\varepsilon(\omega)=1-\frac{\omega_{p}^{2}}{\omega^{2}-i \omega \omega_{\tau}},
$$

where $\omega_{p}$ is the plasma frequency and $\omega_{\tau}$ is the damping frequency. This expression takes the metallic losses through its description into account. Drude parameters used in the calculations for copper are $\omega_{p} / 2 \pi=1914 \mathrm{THz}, \omega_{\tau} / 2 \pi=8.34 \mathrm{THz}[\underline{43}]$.

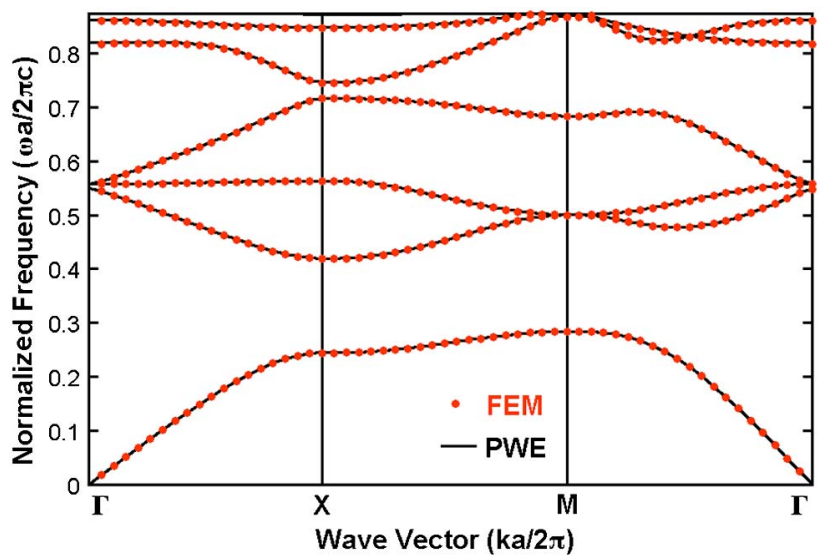

Fig. 3. TM band structure of $2 \mathrm{D}$ photonic crystal in square lattice of silicon rods in air, radius $r=0.2 a$. Solid black lines represent the bands calculated using the PWE method. The bands calculated using FEM are represented with red dots.

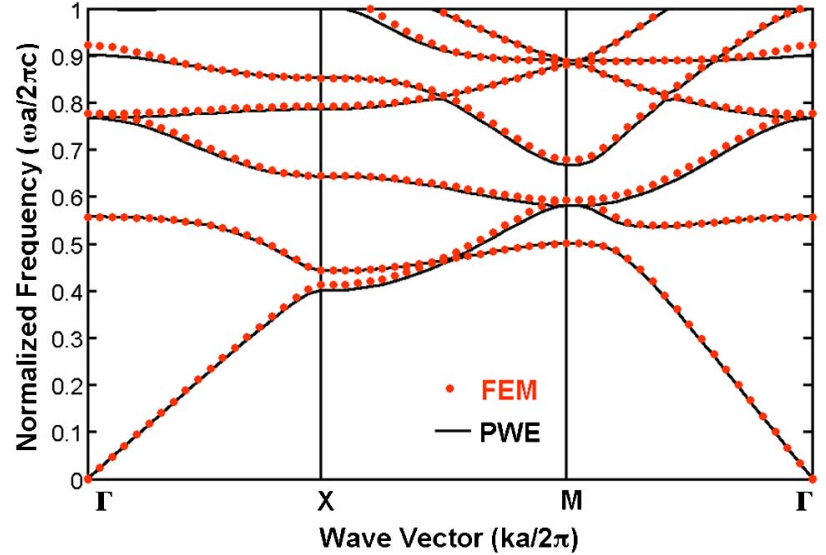

Fig. 4. TE band structure of $2 \mathrm{D}$ photonic crystal in square lattice of silicon rods in air, radius $r=0.2 a$. Solid black lines represent the bands calculated with the PWE method. The bands calculated with FEM are represented with red dots.

The photonic band structure of a system consisting of a square lattice array of copper cylinders embedded in air is calculated. The radius of the rods is $r=0.2 a$, where lattice pattern, $a$, is set at $50 \mu \mathrm{m}$.

The calculated photonic band structures are plotted along high-symmetry directions $\Gamma, X$, and $M$ as depicted in Figs. 5 and 6 for TM and TE modes, respectively. The high-symmetry points are set as $\Gamma(0,0), X(\pi / a, 0)$, and $M(\pi / a, \pi / a)$ from center to near and far edge of the Brillouin zone. In the band gap diagram, photonic bands with the lowest frequencies within $\mathrm{THz}$ range are drawn.

There are two band gaps appearing for metallic photonic crystals in square lattice pattern for TM mode within the $\mathrm{THz}$ range, as seen in the shaded areas marked in Fig. 5. In contrast to the case of dielectric photonic crystals, there is a cutoff frequency and the metallic photonic crystal exhibits rather large photonic band gaps. The frequency range between zero frequency and the cutoff frequency defines the first band gap. The second band gap is

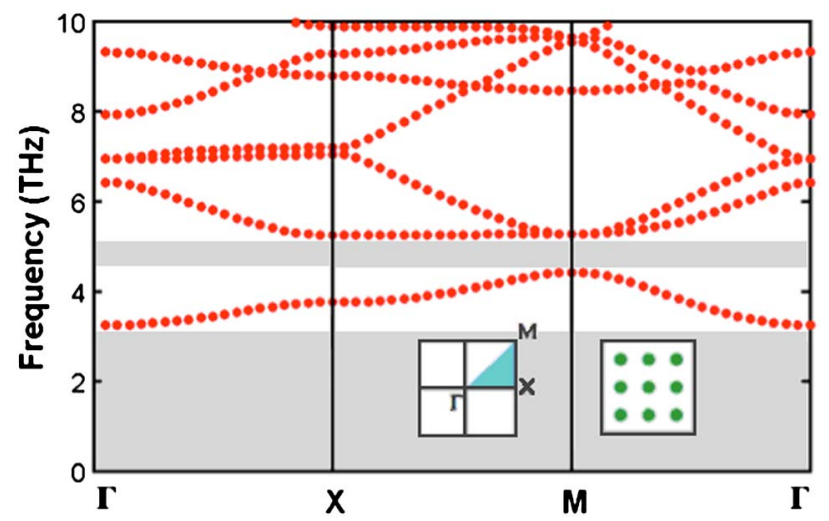

Fig. 5. Photonic band structure of a square lattice of metal cylinders in air for TM mode. The photonic crystal is characterized by a $50 \mu \mathrm{m}$ lattice period in square lattice pattern with a radius of $0.2 a$. The left inset shows the high symmetry points at the corners of the irreducible Brillouin zone; the right inset shows the square lattice pattern. 


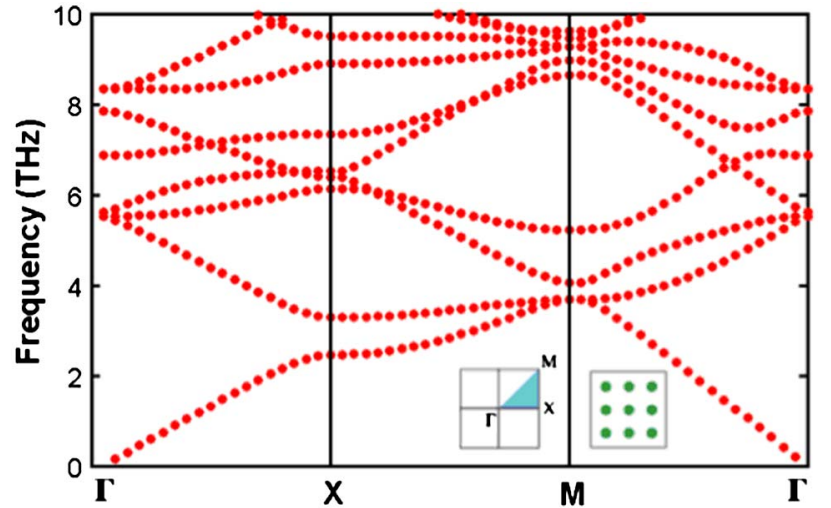

Fig. 6. Photonic band structure of a square lattice of metal cylinders in air for TE mode. The photonic crystal is characterized by a $50 \mu \mathrm{m}$ lattice period in square lattice pattern with a radius of $0.2 a$. The left inset shows the high-symmetry points at the corners of the irreducible Brillouin zone; the right inset shows the square lattice pattern.

located between the maximum of the first band and the minimum of the second band. No wave can propagate through the structure for frequencies falling within the photonic band gap, as opposed to the pass band of the structure where it becomes transparent to the wave propagation.

The first band gap is wider, extending from 0 to $3.244 \mathrm{THz}$, and the second band gap is between 4.413 and $5.242 \mathrm{THz}$. We have represented the frequencies without any normalization in the case of metallic materials; however, these ranges correspond to $0-0.5407(\omega a / 2 \pi c)$ and $0.734-0.8778$ $(\omega a / 2 \pi c)$, respectively, in terms of normalized frequencies. The region between these two bands, 3.244-4.414 THz, is the pass band of the crystal. As can be seen in Fig. 6 , there is no band gap for TE mode. Moreover, for TE mode, unlike TM mode, metallic photonic crystals do not possess a nonzero cutoff frequency. These results are in good agreement with previously published results $[24,31,48]$.

Figure 7 shows the electric field distribution of TM eigenmodes of the six lowest photonic bands of a

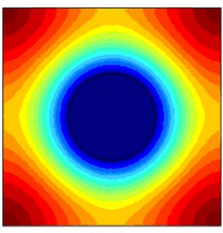

(a)

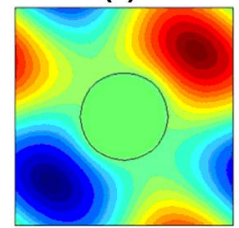

(d)

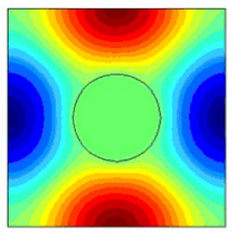

(b)

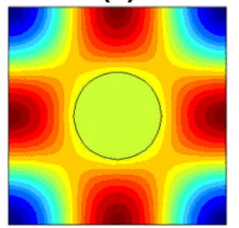

(e)

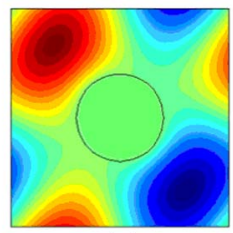

(c)

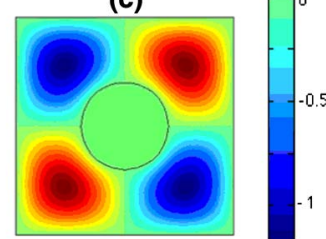

(f)
Fig. 7. Electric field distribution of first six eigenmodes at $\Gamma$ point of a square lattice of metallic circular cylinders for TM mode. In the figures, the maximum of electric field is normalized to unity. square lattice of metallic cylinders at the $\Gamma$ point. The E-field is plotted for the modes from lowest to highest frequency, labeled as (a) $3.244 \mathrm{THz}$, (b) $6.415 \mathrm{THz}$, (c) $6.933 \mathrm{THz}$, (d) $6.933 \mathrm{THz}$, (e) $7.918 \mathrm{THz}$, and (f) $9.308 \mathrm{THz}$.

As a result of spatial symmetry of the lattice structure, all the eigenmodes are symmetric. The first mode [Fig. 7(a)] corresponds to the cutoff frequency, and therefore most of the energy is concentrated in the metallic lattice unlike the other modes shown. The cutoff corresponds to the lowest mode of the $\Gamma$ point in $k$ space. The third and fourth modes appear at the same frequency and their electric field distributions have the same field distribution with mirror symmetry.

The complex eigenvalue problem is now solved for wave vector $k$, for a given frequency $\omega$ in the unit cell of triangular lattice array of copper cylinders embedded in air. The unit cell is indicated by the black frame in Fig. 2. In triangular lattice pattern, the metallic cylinders are arranged periodically; the separation between rods is equal to the lattice constant, $a$, in the $\Gamma-K$ direction, while rods are $\sqrt{3 a}$ apart from each other in the $\Gamma-M$ direction.

The calculated photonic band structures for triangular lattice pattern are plotted along highsymmetry directions $\Gamma, M$, and $K$ as depicted in Figs. $\underline{8}$ and 9 for TM and TE modes, respectively. In the band gap diagrams, the lowest frequency bands in $\mathrm{THz}$ range are drawn. The high-symmetry points $\Gamma, M$, and $K$ from center to near and far edge of the Brillouin zone are set as in Table 1.

The band gap diagram depicted in Fig. 8 shows that there are two band gaps for metallic photonic crystals. Similar to the square lattice case for TM mode, a cutoff frequency is observed. The first band gap is wider than the second one and extends from 0 to $3.764 \mathrm{THz}$, while the second band is very narrow and spans from 7.019 to $7.157 \mathrm{THz}$, corresponding to $0-0.673$ and $1.1698-1.928$ in normalized frequency, respectively. In the band gap diagram for TE mode,

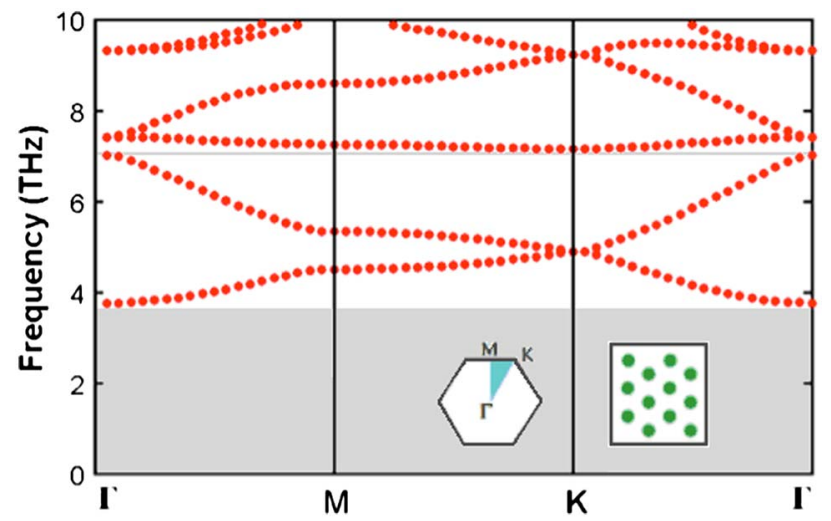

Fig. 8. Photonic band structure of a triangular lattice of metal cylinders in air for TM mode. The photonic crystal is characterized by a $50 \mu \mathrm{m}$ lattice period in triangular lattice pattern with a radius of $0.2 a$. The left inset shows the high-symmetry points at the corners of the irreducible Brillouin zone; the right inset shows the triangular lattice pattern. 


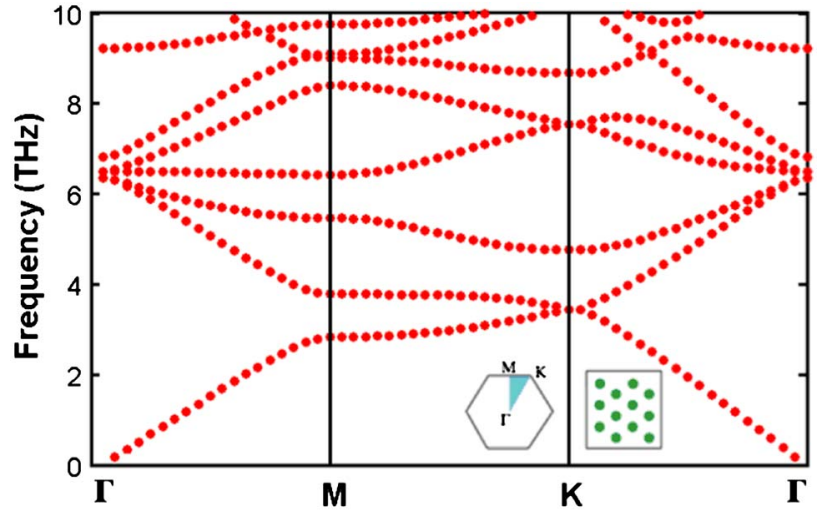

Fig. 9. Photonic band structure of a triangular lattice of metal cylinders in air for TE mode. The photonic crystal is characterized by a $50 \mu \mathrm{m}$ lattice period in triangular lattice pattern with a radius of $0.2 a$. The left inset shows the high-symmetry points at the corners of the irreducible Brillouin zone; the right inset shows the triangular lattice pattern.

neither band gap nor cutoff frequency is observed, as shown in Fig. 9. The lowest frequency bands tend to zero at $\Gamma$ point.

Figure 10 shows the electric field distribution of eigenmodes of the six lowest photonic bands of a triangular array of metallic cylinders at the $\Gamma$ point. The E-field is plotted for the modes from lowest to highest frequency as (a) $3.764 \mathrm{THz}$, (b) $7.019 \mathrm{THz}$, (c) $7.415 \mathrm{THz}$, (d) $7.415 \mathrm{THz}$, (e) $9.313 \mathrm{THz}$, and (f) $9.313 \mathrm{THz}$. Similar to the square lattice eigenmodes, most of the electromagnetic field is concentrated between the metallic cylinders. As can be seen from the eigenmodes in the figure, a triangular lattice pattern supports symmetric (even) and antisymmetric (odd) modes. Antisymmetric modes appear for higher eigenfrequencies.

We may distinguish the parity of modes, whether even or odd, from the electric field distribution of the bands. This helps in understanding the transmission characteristics of the metallic lattice structure. Since only the TM polarization presents a band gap, we are using this property to study the transmission spectra of four layers of copper cylinders in a square lattice pattern.

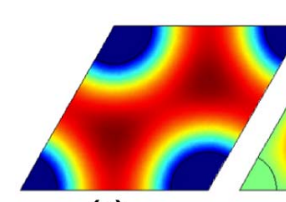

(a)

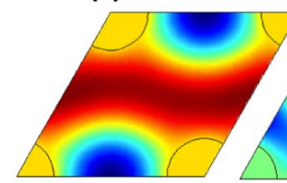

(d)

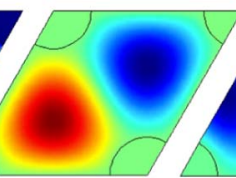

(b)

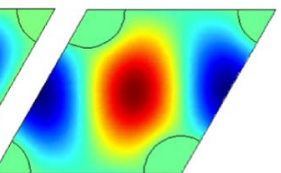

(c)

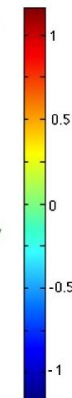

(e) (n)

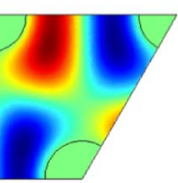

(f)

Fig. 10. Electric field distribution of first six eigenmodes at $\Gamma$ point of a triangular lattice of metallic circular cylinders for TM mode. In the figures, the maximum of electric field is normalized to unity.

\section{Transmission Spectrum Analysis}

Transmission and filtering characteristics of $2 \mathrm{D}$ metallic photonic crystals embedded in parallel plate waveguide have been studied experimentally and compared with simulation results achieved by TMM results in Zhao and Grischkowsky [21]. In the study, five columns of gold-coated SU-8 polymer cylinders embedded in air, in square lattice, with lattice constant of $160 \mu \mathrm{m}$, rod radius $r=0.22 a$, and height of $80 \mu \mathrm{m}$ have been exposed to a signal in the range of $0.5-3 \mathrm{THz}$. A recent study has been conducted on the $\mathrm{THz}$ transmission properties of $2 \mathrm{D}$ metallic photonic crystals in square lattice with control of air gap between the parallel metal plates and the tunability of the stop bands using FDTD method and dispersion relation through its phase in the $\mathrm{THz}$ range up to $2.5 \mathrm{THz}$ [49].

In this section, we present a series of simulations in order to obtain transmission characteristics of $\mathrm{THz}$ wave propagation within $2 \mathrm{D}$ metallic photonic crystals in square and triangular lattice patterns scanning the whole $\mathrm{THz}$ range. The $\mathrm{THz}$ transmission and dispersion relations of $2 \mathrm{D}$ metallic photonic crystals are investigated in detail and a comparison will be drawn in order to understand the propagation characteristics. As no band gap appears for metallic photonic structures in TE mode, transmission spectra is calculated only for TM mode.

Power transmission is defined as the ratio of power flowing through the output boundary on the incident power at the input boundary. Nonreflecting boundary conditions are used in order to prevent any reflection which could affect the power measurements at both locations.

Transmission spectrum of a $2 \mathrm{D}$ metallic photonic crystal structure consisting of copper rods is calculated over a geometry shown in Fig. 11. The direction of wave at a normal incident corresponds to the $\Gamma-X$ direction in crystal.

Figure 12 shows (a) the transmission spectra of 4 layers of rods and (b) a band gap diagram of a square lattice structure. On the one hand, transmission occurs only for eigenvectors in the direction of the incident radiation, the $\Gamma-X$ direction. On the other hand, within the band gap frequencies, the structure

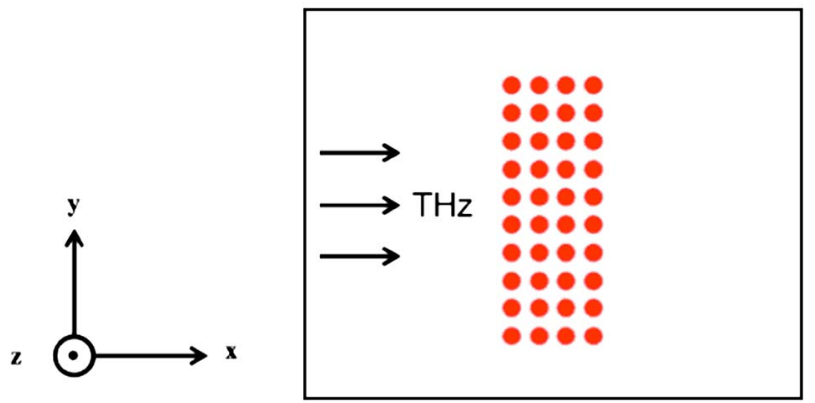

Fig. 11. Schematic illustration of the geometry used in calculations. Red circles correspond to metallic cylinders. Nonreflecting boundary conditions are used to surround computational area in order to prevent reflections. 
(a)

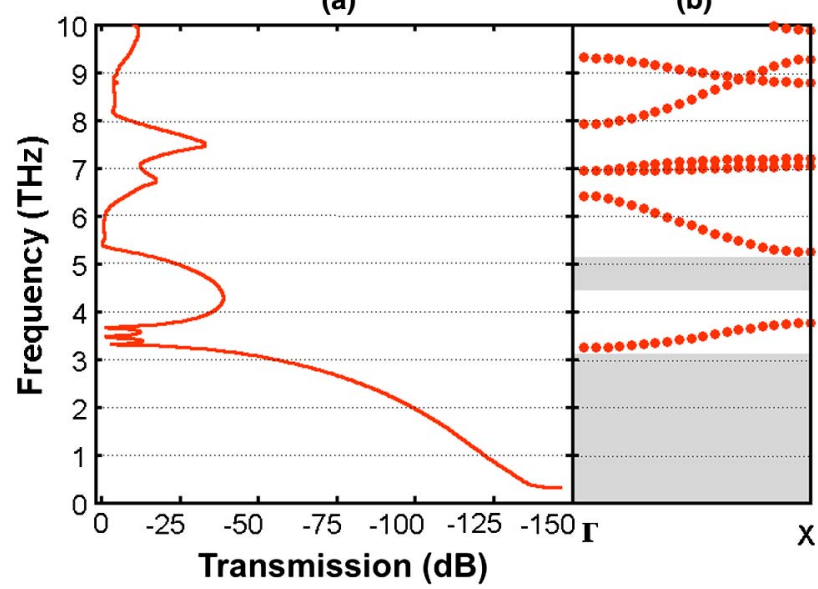

Fig. 12. (a) Transmission spectra are calculated for 4 layers of square lattice structure in the $\Gamma-X$ direction. (b) Photonic band structure of a square lattice of metallic rods in the $\Gamma-X$ direction. The rods' radius is $r=0.2 a$ where $a=50 \mu \mathrm{m}$. Shaded areas represent common photonic band gaps for any crystal direction.

is reflective; therefore, the transmission of incident wave to the output side of the crystal structure is drastically suppressed. The position of transmission dips follows the band gap diagram. In the first band gap, between 0 and $3.244 \mathrm{THz}$, the transmission is as low as $-150 \mathrm{~dB}$, indicating the cutoff frequency. The first pass band is between 3.244 and $3.751 \mathrm{THz}$. The frequency corresponds to the pass bands of the square lattice structure. The second band gap is between 3.751 and $5.236 \mathrm{THz}$; there are also two other small band gaps between $6.415-6.933 \mathrm{THz}$ and 7.188-7.918 THz. It is clear that the corresponding positions of the dips and peaks of the transmission results show excellent agreement with the band gap diagram.

The position of dips corresponds to the point on the $\Gamma$ direction as the wave is at normal incidence. It also clearly shows the existence of cutoff frequency between zero frequency and a certain cutoff value where there is no propagation.

Figure 13 gives an illustration of the electric field propagation at normal incidence to square lattice crystal for two frequencies at 3 and $3.5 \mathrm{THz}$. If the frequency is within a photonic band gap as at

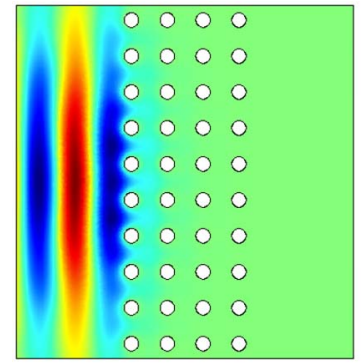

(a)

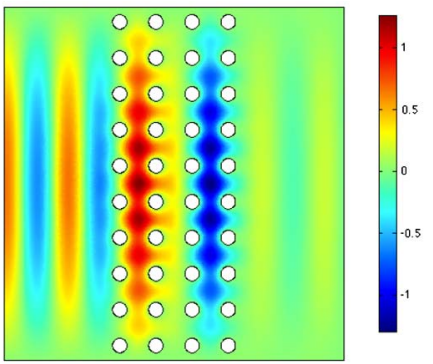

(b)
Fig. 13. Electric field distribution metallic photonic structure in a square lattice array with rod radius of $0.2 a$, where lattice constant $a=50 \mu \mathrm{m}$ at (a) $3 \mathrm{THz}$ and (b) $3.5 \mathrm{THz}$.
$3 \mathrm{THz}$, the E field rapidly decays inside the crystal; otherwise, it propagates through the array as depicted for $3.5 \mathrm{THz}$.

Transmission spectrum of metallic photonic crystal in triangular pattern is calculated along the $\Gamma-M$ and $\Gamma-K$ directions for TM mode. Figure 14(a) shows the transmission spectra of triangular lattice array consisting of 5 layers with lattice constant of $50 \mu \mathrm{m}$ and rod radius of $0.2 a$, while Fig. 14(b) shows the band gap diagram of triangular lattice along high symmetry points.

The band gap diagram represents the 6 lowest bands for metallic cylinders in triangular lattice array in the $\mathrm{THz}$ range. In the band gap diagram, the shaded areas show the band gap for any crystal direction. The transmission is calculated for $\Gamma-M$ and $\Gamma-K$ directions, and it exhibits the band characteristics in the given direction. The position of transmission dips and peaks follows the same pattern with the band gap diagram. The frequencies at the band gap edge indicated by the high symmetry points coincide with transmission spectrum of corresponding propagation direction.

It can be seen from the transmission spectra that transmission occurs only at the frequencies where there are eigenmodes with wave vectors in the direction of the incident radiation. However, as mentioned earlier, not all the modes for this structure are symmetric. For instance, the third mode in the $\Gamma-M$ direction and second and fourth modes in the $\Gamma-K$ direction are antisymmetric. The symmetric (even) modes are represented by red dots while antisymmetric (odd) modes are represented by red open dots. When two modes are intersecting at the highsymmetry points, mode crossing occurs. The two modes intersecting at a symmetry point have a different sign of group velocities. One of the modes is symmetric and the other is antisymmetric. The symmetric input field can only couple with symmetric modes. The uncoupled modes lead to low transmission [50]. (a)

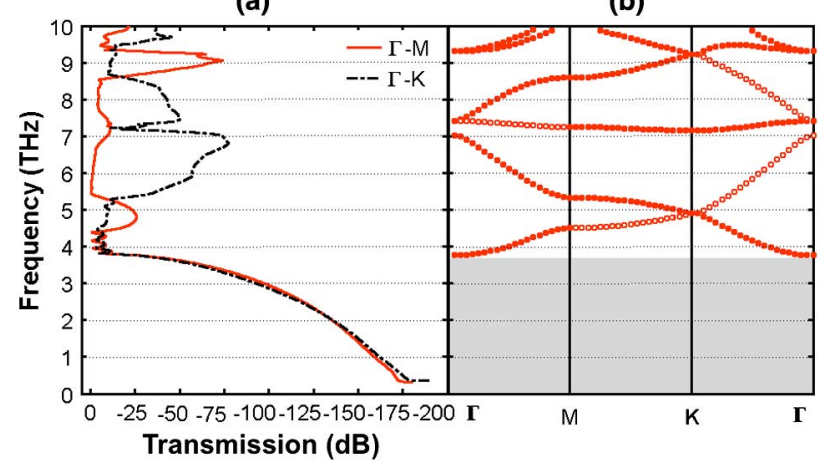

Fig. 14. (a) Transmission spectra are calculated for five layers of triangular lattice structure in the $\Gamma-M$ and $\Gamma-K$ directions. (b) Photonic band structure of a triangular lattice of metallic rods in TM mode. The rod radius is $r=0.2 a$, where $a=50 \mu \mathrm{m}$. Shaded areas represent common photonic band gaps in any crystal direction for TM mode. 

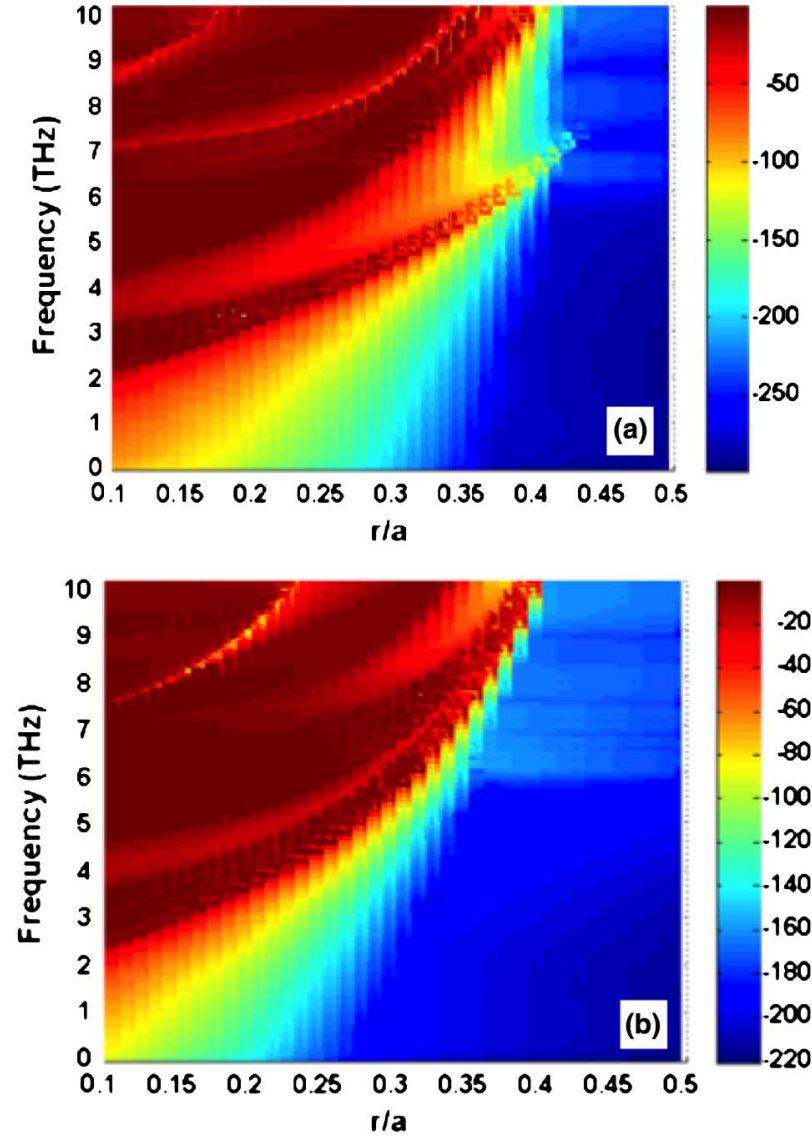

Fig. 15. Photonic band gap map of metallic photonic crystal structure in (a) square lattice pattern and (b) triangular lattice pattern.

The photonic band structure and the transmission spectra figures match, positions of the dips and peaks of the transmission spectra can be followed from the dispersion diagram in Fig. 14(b). In both the $\Gamma-M$ and $\Gamma-K$ directions, the first band gap ranges between 0 and $3.764 \mathrm{THz}$ with a transmission as low as $-175 \mathrm{~dB}$. The frequency ranges where there is a wave transmission correspond to the pass bands of triangular lattice structure for the given direction. In the $\Gamma-M$ direction, the first pass band is between 3.764 and $4.507 \mathrm{THz}$. The second band gap is between 4.507 and $5.311 \mathrm{THz}$. There are also two other small band gaps between 7.019$7.253 \mathrm{THz}$ and 8.594-9.313 $\mathrm{THz}$. In the range between 7.253 and $7.415 \mathrm{THz}$, the low transmission is due to the different symmetry of the modes involved. In the $\Gamma-K$ direction, the first pass band is between 3.764 and $4.901 \mathrm{THz}$. Even though modes appear in the 4.901-7.019 THz and 7.157-9.288 THz ranges, there are dips in transmission spectrum since input field cannot couple with these antisymmetric modes in the $\Gamma-K$ direction. The peak between 7.019 and $7.157 \mathrm{THz}$ in the transmission figure corresponds to the small pass band in the dispersion figure.

The photonic band gap map of the 2D metallic photonic crystals is obtained for various values of rod radii $r / a$ by calculating the corresponding transmission spectrum instead of computation of the band structure as usually done. The contour maps seen in Figs. 15(a) and 15(b) show the band gap maps obtained through transmission spectra for square lattice along the $\Gamma-X$ direction and triangular lattice along the $\Gamma-M$ direction, respectively, by varying the radii from $0.1 a$ to $0.49 a$. The color scales on the right-hand side of the each figure show transmission levels in $\mathrm{dB}$ while frequencies are shown on the $y$ axis of the contour figures. The band gap maps in Figs. 15(a) and 15(b) show good agreement with the band gap maps studied previously [51]. As can be seen from these figures, for larger rod sizes, transmission through metallic structure is very low due to high reflection from the structure. In both square and triangular lattice for $r / a$ ratio over 0.4 , the structures are reflective for whole $\mathrm{THz}$ frequency range where transmission is lower than $-120 \mathrm{~dB}$. Using these metallic structures, high-reflectivity walls can be built at $\mathrm{THz}$ frequencies.

\section{Conclusion}

In summary, FEM was employed to investigate $\mathrm{THz}$ transmission and dispersion characteristics of 2D metallic photonic crystals arranged in square and triangular lattice patterns for TE and TM modes in great detail. In the calculations, material dispersion and frequency dependency were taken into account. The modal field symmetry characteristics have also been discussed. A FEM model was set up to calculate band gap diagram of metallic photonic crystals for both square and triangular lattice arrays.

A strong correlation is found between the key features, i.e., positions of the dips and peaks of a transmission spectra and the corresponding photonic band structure. The comparison showed a qualitative consistency between the band gap diagram results and the transmission spectra. The position and width of the photonic band gaps is well reproduced in transmission spectra, but the photonic band gap analysis helps in understanding the location and the origin of the dip in the spectra.

\section{References}

1. P. H. Siegel, "Terahertz technology," IEEE Trans. Microw. Theory Tech. 50, 910-928 (2002).

2. M. Tonouchi, "Cutting-edge terahertz technology," Nat. Photonics 1, 97-105 (2007).

3. R. E. Miles, P. Harrison, and D. Lippens, Terahertz Sources and Systems (Springer, 2001).

4. J. D. Joannopoulos, S. G. Johnson, J. N. Winn, and R. D. Meade, Photonic Crystals: Molding the Flow of Light, 2nd ed. (Princeton University, 2008).

5. C. Lin, C. Chen, G. Schneider, P. Yao, S. Shi, A. Sharkawy, and D. Prather, "Wavelength scale terahertz two-dimensional photonic crystal waveguides," Opt. Express 12, 5723-5728 (2004).

6. Z. Li, Y. Zhang, and B. Li, "Terahertz photonic crystal switch in silicon based on self-imaging principle," Opt. Express 14, 3887-3892 (2006).

7. H. Nemec, P. Kuzel, L. Duvillaret, A. Pashkin, M. Dressel, and M. T. Sebastian, "Highly tunable photonic crystal filter for the terahertz range," Opt. Lett. 30, 549-551 (2005).

8. T.-B. Yu, M.-H. Wang, X.-Q. Jiang, Q.-H. Liao, and J.-Y. Yang, "Ultracompact and wideband power splitter based on triple 
photonic crystal waveguides directional coupler," J. Opt. A 9, 37-42 (2007).

9. E. R. Brown and O. B. McMahon, "Large electromagnetic stop bands in metallodielectric photonic crystals," Appl. Phys. Lett. 67, 2138-2140 (1995).

10. S. Fan, P. R. Villeneuve, and J. D. Joannopoulos, "Large omnidirectional band gaps in metallodielectric photonic crystals," Phys. Rev. B 54, 11245-11251 (1996).

11. Z.-Y. Li, I. El-Kady, K.-M. Ho, S. Y. Lin, and J. G. Fleming, "Photonic band gap effect in layer-by-layer metallic photonic crystals," J. Appl. Phys. 93, 38-42 (2003).

12. A. R. McGurn and A. A. Maradudin, "Photonic band structures of two- and three-dimensional periodic metal or semiconductor arrays," Phys. Rev. B 48, 17576-17579 (1993).

13. D. R. Smith, S. Schultz, N. Kroll, M. Sigalas, K. M. Ho, and C. M. Soukoulis, "Experimental and theoretical results for a two-dimensional metal photonic band-gap cavity," Appl. Phys. Lett., 65, 645-647, (1994).

14. M. M. Sigalas, C. T. Chan, K. M. Ho, and C. M. Soukoulis, "Metallic photonic band-gap materials," Phys. Rev. B 52, 11744-11751 (1995).

15. E. Ozbay and B. Temelkuran, "Reflection properties and defect formation in photonic crystals," Appl. Phys. Lett. 69 743-745 (1996)

16. F. Gadot, A. Lustrac, J. M. De Lourtioz, T. Brillat, A. Ammouche, and E. Akmansoy, "High-transmission defect modes in two-dimensional metallic photonic crystals," J. Appl. Phys. 85, 8499-8501 (1999).

17. M. Bayindir, E. Cubukcu, I. Bulu, T. Tut, E. Ozbay, and C. M. Soukoulis, "Photonic band gaps, defect characteristics, and waveguiding in two-dimensional disordered dielectric and metallic photonic crystals," Phys. Rev. B 64, 195113 (2001).

18. C. Jin, B. Cheng, Z. Li, D. Zhang, L. M. Li, and Z. Q. Zhang, "Two dimensional metallic photonic crystal in the $\mathrm{THz}$ range," Opt. Commun. 166, 9-13 (1999).

19. S. W. Wang, W. Lu, X. S. Chen, Z. F. Li, X. C. Shen, and W. Wen, "Two-dimensional photonic crystal at $\mathrm{THz}$ frequencies constructed by metal-coated cylinders," J. Appl. Phys. 93, 9401-9403 (2003).

20. N. Katsarakis, M. Bender, L. Singleton, G. Kiriakidis, and C. M. Soukoulis, "Two-dimensional metallic photonic bandgap crystals fabricated by LIGA," Microsyst. Technol. 8, 74-77 (2002).

21. Y. Zhao and D. R. Grischkowsky, "2-D terahertz metallic photonic crystals in parallel-plate waveguides," IEEE Trans. Microw. Theory Tech. 55, 656-663 (2007).

22. A. L. Bingham and D. R. Grischkowsky, "Terahertz 2-D photonic crystal waveguides," IEEE Microw. Wirel. Compon. Lett. 18, 428-430 (2008).

23. E. Degirmenci, F. Surre, S. Philippe, R. Maldonado-Basilio, and P. Landais, "Improved bend waveguide design for terahertz transmission," IEEE Trans. Terahertz Sci. Technol. 2, 137-143 (2012).

24. V. Kuzmiak, A. A. Maradudin, and F. Pincemin, "Photonic band structures of two-dimensional systems containing metallic components," Phys. Rev. B 50, 16835 (1994).

25. S. Shi, C. Chen, and D. W. Prather, "Revised plane wave method for dispersive material and its application to band structure calculations of photonic crystal slabs," Appl. Phys. Lett. 86, 043104 (2005).

26. A. Nicorovici, R. C. McPhedran, and L. C. Botten, "Photonic band gaps for arrays of perfectly conducting cylinders," Phys. Rev. E 52, 1135-1145 (1995)

27. K. Sakoda, N. Kawai, T. Ito, A. Chutinan, S. Noda, T. Mitsuyu, and K. Hirao, "Photonic bands of metallic systems. I. Principle of calculation and accuracy," Phys. Rev. B 64, 045116 (2001).

28. T. Ito and K. Sakoda, "Photonic bands of metallic systems. II. Features of surface plasmon polaritons," Phys. Rev. B 64, 045117 (2001).

29. O. Takayama and M. Cada, "Two-dimensional metallodielectric photonic crystals embedded in anodic porous alumina for optical wavelengths," Appl. Phys. Lett. 85, 1311-1313 (2004).
30. M. Qiu and S. He, "A nonorthogonal finite-difference timedomain method for computing the band structure of a two-dimensional photonic crystal with dielectric and metallic inclusions,” J. Appl. Phys. 87, 8268-8275 (2000).

31. E. I. Smirnova, C. Chen, M. A. Shapiro, J. R. Sirigiri, and R. J. Temkin, "Simulation of photonic band gaps in metal rod lattices for microwave applications," J. Appl. Phys. 91, 960-968 (2002).

32. A. Raman and S. Fan, "Photonic band structure of dispersive meta-materials formulated as a Hermitian eigenvalue problem," Phys. Rev. Lett. 104, 087401 (2010).

33. E. Moreno, D. Erni, and C. Hafner, "Band structure computations of metallic photonic crystals with the multiple multipole method," Phys. Rev. B 65, 155120 (2002).

34. H. van der Lem, A. Tip, and A. Moroz, "Band structure of absorptive two-dimensional photonic crystals," J. Opt. Soc. Am. B 20, 1334-1341 (2003).

35. A. Modinos, N. Stefanou, and V. Yannopapas, "Applications of the layer-KKR method to photonic crystals," Opt. Express 8, 197-202 (2001).

36. A. Moroz, "Metallo-dielectric diamond and zinc-blende photonic crystals," Phys. Rev. B 66, 115109 (2002).

37. S. Liu and Z. Lin, "Opening up complete photonic bandgaps in three-dimensional photonic crystals consisting of biaxial dielectric spheres," Phys. Rev. E 73, 066609 (2006).

38. J. Arriaga, A. J. Ward, and J. B. Pendry, "Order-N photonic band structures for metals and other dispersive materials," Phys. Rev. B 59, 1874-1877 (1999).

39. Y. Tsuji and M. Koshiba, "Finite element method using port truncation by perfectly matched layer boundary conditions for optical waveguide discontinuity problems," J. Lightwave Technol. 20, 463-468 (2002).

40. W. J. Kim and J. D. O'Brien, "Optimization of a twodimensional photonic-crystal waveguide branch by simulated annealing and the finite-element method," J. Opt. Soc. Am. B 21, 289-295 (2004).

41. N. Kono and Y. Tsuji, "A novel finite-element method for nonreciprocal magneto-photonic crystal waveguides," J. Lightwave Technol. 22, 1741-1747 (2004).

42. "COMSOL Multiphysics," 2005, www.comsol.com.

43. M. A. Ordal, R. J. Bell, R. W. Alexander, Jr., L. L. Long, and M. R. Querry, "Optical properties of fourteen metals in the infrared and far infrared: $\mathrm{Al}, \mathrm{Co}, \mathrm{Cu}, \mathrm{Au}, \mathrm{Fe}, \mathrm{Pb}, \mathrm{Mo}, \mathrm{Ni}$, Pd, Pt, Ag, Ti, V, and W.," Appl. Opt. 24, 4493-4499 (1985).

44. M. Davanco, Y. Urzhumov, and G. Shvets, "The complex Bloch bands of a $2 \mathrm{D}$ plasmonic crystal displaying isotropic negative refraction," Opt. Express 15, 9681-9691 (2007).

45. B. P. Hiett, J. M. Generowicz, S. J. Cox, M. Molinari, D. H. Beckett, and K. S. Thomas, "Application of finite element methods to photonic crystal modelling," IEE Proc. A Sci. Meas. Technol. 149, 293-296 (2002).

46. C. Fietz, Y. Urzhumov, and G. Shvets, "Complex $k$ band diagrams of 3D metamaterial/photonic crystals," Opt. Express 19, 19027-19041 (2011)

47. T. Yamashita and C. J. Summers, "Evaluation of selfcollimated beams in photonic crystals for optical interconnect," IEEE J. Sel. Areas Commun. 23, 1341-1347 (2005).

48. M. Qiu and S. He, "Guided modes in a two-dimensional metallic photonic crystal waveguide," Phys. Lett. A 266, 425-429 (2000).

49. J. Kitagawa, M. Kodama, S. Koya, Y. Nishifuji, D. Armand, and Y. Kadoya, "THz wave propagation in two-dimensional metallic photonic crystal with mechanically tunable photonic-bands," Opt. Express 20, 17271-17280 (2012).

50. Z. Jian, J. Pearce, and D. M. Mittleman, "Two-dimensional photonic crystal slabs in parallel-plate metal waveguides studied with terahertz time-domain spectroscopy," Semicond. Sci. Technol. 20, S300-S306 (2005).

51. M.-C. Lin and R.-F. Jao, "Finite element analysis of photon density of states for two-dimensional photonic crystals with in-plane light propagation," Opt. Express 15, 207-218 (2007). 